PHYSICAL REVIEW E 93, 059901(E) (2016)

\title{
Erratum: Macroscopic fluctuation theory and first-passage properties of surface diffusion
}

[Phys. Rev. E 93, 020102(R) (2016)]

Baruch Meerson and Arkady Vilenkin

(Received 28 April 2016; published 9 May 2016)

DOI: 10.1103/PhysRevE.93.059901

The extension of our one-dimensional results to higher dimensions, presented in the paragraph around Eq. (33), is incorrect. Equation (33) is correct only at $d=1$. At $d>1$ the variance $\sigma_{d}$ is actually infinite.

We thank N. R. Smith for helping us clarify the origin of this error. 